Revue d'histoire de l'enfance « irrégulière »

Le Temps de l'histoire

Hors-série | 2001

Histoire et justice, panorama de la recherche

\title{
Les centres de recherche universitaires
}

Table ronde

Jacques-Guy Petit, Benoît Garnot, Martine Kaluszynski, Laurence

Dumoulin, Michel Cassan, Michel Porret, Jean-Marie Augustin et

Frédéric Chauvaud

\section{(2) OpenEdition}

Journals

Édition électronique

URL : http://journals.openedition.org/rhei/413

DOI : $10.4000 /$ rhei. 413

ISBN : 978-2-7535-1641-0

ISSN : $1777-540 \mathrm{X}$

Éditeur

Presses universitaires de Rennes

Édition imprimée

Date de publication : 15 novembre 2001

Pagination : $57-87$

ISSN : 1287-2431

Référence électronique

Jacques-Guy Petit, Benoît Garnot, Martine Kaluszynski, Laurence Dumoulin, Michel Cassan, Michel Porret, Jean-Marie Augustin et Frédéric Chauvaud, "Les centres de recherche universitaires », Revue d'histoire de l'enfance « irrégulière » [En ligne], Hors-série | 2001, mis en ligne le 31 mai 2007, consulté le 21 avril 2019. URL : http://journals.openedition.org/rhei/413; DOI : 10.4000/rhei.413 


\section{Les centres de recherche universitaires}

\section{Jacques-Guy Petit}

Historien, directeur de l'Hirés, université d'Angers

Frédéric Chauvaud m'a demandé de présenter l'itinéraire d'un centre de recherche historique, principalement consacré à l'histoire de la justice, dans le cadre d'une faculté de Lettres. Je pense que pour beaucoup de jeunes DEA ou de doctorants, vous allez trouver ici une trajectoire qui peut être proche de ce que vous voyez autour de vous, à Poitiers, en train de se constituer. Ce centre, l'Hirés (centre d'Histoire des régulations et des politiques sociales), existe depuis huit ans dans le Département d'histoire de l'université d'Angers. Équipe d'accueil (UPRES EA), il compte actuellement environ 30 chercheurs et 20 doctorants. Il s'est créé autour des travaux que j'avais réalisés avec d'autres sur l'histoire de la peine, l'histoire de l'enfermement, dans les années 70-80, en collaboration avec beaucoup de chercheurs ou de réseaux qui sont représentés ici aujourd'hui. Il s'inscrit dans une certaine filiation, qui est celle des travaux de Michelle Perrot et de Paris VII, ce qui est une façon d'envisager l'histoire sociale. A cette époque, à ma connaissance, il n'y avait pas de centre de recherche dans les facultés de lettres consacré à l'Histoire de la justice. C'est le moment, en tout cas en histoire contemporaine, où ma génération avait été marquée par les travaux de Michel Foucault, la recherche du fonctionnement de la justice, la façon dont les normes sont produites, dont les procédures sont mises en œuvre jusqu’à l'exécution de la peine, tout ceci permettant de mieux comprendre le fonctionnement réel d'une société globale dans son ensemble. Michel

Jacques-Guy Petit, Benoît Garnot, Martine Kaluszynski, Laurence Dumoulin, Michel Cassan, Michel Porret, Jean-Marie Augustin, Frédéric Chauvaud / pp. 57 à 87 
Foucault l'a bien montré, en particulier pour le siècle des Lumières et le XIXème siècle.

Un centre de recherche dans une faculté des lettres, cela représente des contraintes qui sont à la fois des handicaps, mais aussi des chances, dans la mesure où on est obligé de sortir des sentiers battus. Je vais rappeler quelques-uns de ces handicaps.

- Le premier est de ne pas être des juristes de formation. Nous n'avons pas de formation juridique et jusqu'alors, pour l'essentiel, l'histoire de la justice se faisait dans le cadre des facultés de droit par les spécialistes de l'histoire du droit. A Poitiers, il y avait Bernard Schnapper avec lequel nous avons beaucoup travaillé et aussi Jean-Marie Augustin et d'autres comme J. Poumarède à Toulouse, Ph.-J. Hesse à Nantes, Jean Bart à Dijon, ou Jean-Pierre Royer à Lille. Mais en général les historiens du droit et des facultés de droit, sans nous voir arriver avec jalousie nous n'étions pas en situation de rivalité avec eux- nous ont réservé un accueil négatif.

Je peux dire que, dans beaucoup de nos colloques d'historiens, on invite des historiens du droit. La réciproque ne se fait pas toujours, pour des raisons certainement institutionnelles, très caractéristiques du cas français, ce qui est certainement dommageable. En France, il y a encore beaucoup d'efforts à faire pour sortir de ce manque de mise en synergie de réseaux qui travaillent en fait, avec des instruments différents, sur le même objet : la société et le fonctionnement de la justice dans la société.

C'était un handicap parce que cela suppose que l'on acquiert le minimum. C'est une des raisons, pour lesquelles on a surtout travaillé, nous les historiens des facultés des Lettres, d'abord sur le droit pénal, sur la justice pénale dans la mesure où la formation juridique dans ce domaine est certainement plus facile d'accès que l'ensemble des procédures civiles, du droit civil et du jugement civil.

- L'autre handicap, dans ces facultés de lettres, tient au fait que nous avons beaucoup de charges d'enseignement. Nous avons un centre de recherche reconnu par le ministère de l'Enseignement et de la Recherche, donc avec des crédits, mais nous n'avons pas de permanents. Nous sommes tous des enseignants-chercheurs, nous sommes vingt-trois à la 
faculté d'Histoire d'Angers avec 900 étudiants. Nous enseignons du deug à l'agrégation, en passant par les maitrises (140 par an), un enseignement de licence, un enseignement de DEA, etc. Nous avons chacun à faire nos recherches entre la correction de deux cents copies, la préparation de quatre heures de cours en amphi, etc. C'est une contrainte qui se voit en particulier lorsque les grands organismes nous envoient des appels d'offres. Nous ne pouvons répondre, faute de permanent de la recherche que de façon beaucoup moins rapide, beaucoup moins efficace. Ce sont quelques-unes de nos contraintes.

Ces contraintes qui sont en partie des handicaps, sont aussi des chances et même surtout des chances puisque dans une faculté des lettres, par beaucoup d'aspects, nous sommes des généralistes. Depuis quelques années, j'enseigne l'histoire de la justice en maîtrise. Mais jusqu'à présent mes enseignements étaient très divers, rythmés par les programmes des concours, comme pour la plupart d'entre nous. On enseigne ainsi l'histoire de la France, l'histoire des États-Unis, l'histoire de l'ancienne URSS, l'histoire sociale, l'histoire politique, etc.

Tout compte fait, cela est utile pour la façon de concevoir la justice, car c'est une façon de sortir de notre spécialité étroite. Nous avons une vision nécessairement large de la place de la justice et même de l'histoire dans la société.

D'autre part, nous avons aussi une dynamique de groupe. Nous avons eu la chance de monter un centre de recherche qui a travaillé de façon trans-périodes, aussi bien avec les historiens de l'antiquité, que du moyenâge, de l'époque moderne, que de l'époque contemporaine. Nous avons réussi à nous motiver, à côté de nos recherches personnelles qui peuvent continuer, à faire un travail d'équipe autour de la justice mais aussi plus largement sur les régulations sociales. Il y a donc deux aspects complémentaires : les politiques sociales et le fonctionnement de la justice.

La particularité de l'université, c'est de former des jeunes chercheurs. Nous formons à la recherche dans le cadre de DEA et dans le cadre de thèses. Nous avons certes des thèses et des DEA qui sont tout à fait en 
dehors de l'histoire de la justice. Mais on peut aussi trouver de temps en temps, parfois difficilement, des étudiants qui acceptent de se consacrer à l'histoire de la justice. Nous sommes en train de monter un DEA sur les régulations sociales, avec les juristes, avec la géographie sociale et les historiens, ce qui va certainement élargir encore notre palette.

D'autre part, nous avons monté à Angers une formation d'archivistique qui va jusqu'au DESS. Et là nous avons trouvé dans le cadre des stages de cinquième année de DESS, et même dans le cadre des mầtrises, des étudiants qui se consacrent aux archives de la justice.

$\mathrm{Si}$ nous formons environ douze étudiants qui sont reçus au Capes chaque année, un agrégé tous les deux ans peut-être, nous avons vingt étudiants formés en archivistique. La plupart se sont formés sur l'histoire de la justice puisque, pour leur stage, ces étudiants trouvent du travail dans le domaine de l'archivistique publique ou privée en particulier dans les entreprises, aussi bien à l'étranger qu'en France. En prolongement de ces travaux, nous avons organisé à Angers (avec la collaboration de F. Chauvaud) un colloque international sur les rapports entre l'histoire contemporaine, la justice et les archives, qui a été publié dans la collection des Archives nationales.

Nous avons inventé une sorte de maîtrise mixte, acceptée par l'université, avec un travail de classement dans un fond d'archivistique et un travail historique, donc en deux parties. Nous avons inventé ce système qui fonctionne très bien et qui nous permet, à Angers comme dans toute la France, d'envoyer des stagiaires qui classent des fonds d'archives jusqu'à Montréal, en particulier dans le domaine de la police, de la justice, de la pénitentiaire.

Autre synergie, avec la délinquance et la justice des mineurs, dont JeanJacques Yvorel a beaucoup parlé tout à l'heure. Le réseau des équipes et des institutions qui a travaillé sur les mineurs et la justice dans le domaine du privé est venu chez nous demander de déposer - dans la bibliothèque universitaire - un fond d'archives extrêmement important. Nous sommes déjà arrivés à deux kilomètres et cela s'agrandit presque d'un kilomètre tous les deux ans. C'est le Cnaes (le Centre national des archives et de l'histoire de l'éducation surveillée ou spécialisée) qui a crée le Capea 
(Centre des archives de la protection de l'enfance et de l'adolescence), à Angers, en liaison avec l'Hirés, notre Centre de recherche.

Nous sommes aussi de plus en plus ouverts aux dimensions internationales, en collaborant avec des universités et centres d'Amérique du Nord (Canada surtout) et des Pays d'Europe centrale et orientale (Hongrie, Roumanie). Nous appartenons au réseau Gern (Groupe européen de recherche sur les normativités).

Enfin deux domaines sur lesquels nous travaillons actuellement : les justices de paix, sachant que la justice civile qui est très représentée dans les justices de paix a été relativement peu étudiée. Cette justice de proximité, qui est en cours de classement dans les archives départementales pour le XIXème siècle est encore insuffisamment exploitée pour l'histoire sociale.

Et puis, dernier projet, un colloque international sur la différence des sexes et la justice pénale, c'est-à-dire sur la façon dont la justice, du début de la procédure jusqu'à l'exécution de la peine, traite de façon différenciée les hommes ou les femmes. Voilà quelques-unes de nos perspectives actuelles.

\section{Benoît Garnot}

Historien, directeur du CEH - devenu depuis une des composantes du Centre Georges Chevrier-, université de Dijon

Je ne reviens pas sur ce que vient de dire Jacques-Guy Petit sur les conditions de travail de centres comme le sien ou le mien, qui sont assez comparables. Je vais me contenter de vous présenter ce Centre d'études historiques sur la criminalité et les déviances ou plus simplement $\mathrm{CEH}$, qui est une équipe que j’ai créée en 1991. A cette époque, elle avait le statut de "jeune équipe". Elle appartient l'UMR/CNRS 5605, ce qui n'empêche pas que la plupart d'entre nous soyons nous aussi des enseignants-chercheurs. Il y a quand même quelques chercheurs à temps plein, dont Jean-Claude Farcy qui vient de nous rejoindre. Nous participons aussi de façon importante au DEA Ordre et Désordre, qui est basé à Dijon. 
Je vais me contenter de vous donner les résultats concrets du travail de cette équipe depuis sa création il y a un peu plus de huit ans. Cela se précise dans plusieurs directions. D'abord la réalisation de colloques tous les deux ans qui sont des colloques qui se veulent diachroniques, transversaux au point de vue chronologique, qui vont de l'Antiquité jusqu'à l'époque contemporaine - ce qui n'est pas si courant - qui permettent de réunir un grand nombre des spécialistes des problèmes de la criminalité, de l'histoire de la criminalité de France et de l'étranger, du moins de pays limitrophes.

Ces colloques sont publiés en général six mois après. Ce sont des volumes qui font entre 500 et 600 pages, qui sont disponibles dans les bibliothèques et notamment ici. Le premier portait sur Histoire et criminalité, de l'Antiquité au XXème siècle, le second sur Ordre moral et délinquance, le troisième sur L'infra-judiciaire, le quatrième sur La petite délinquance. Le cinquième sur Les victimes, de l'Antiquité au XXème siècle, que nous avons tenu en octobre dernier et qui vient d'être publié.

Le prochain, en 2001, portera sur Les témoins de l'Antiquité au XXème siècle. D'ailleurs si vous avez des sujets de communication à me proposer, j'en serais ravi puisque je cherche toujours à associer des jeunes chercheurs à des chercheurs plus confirmés dans ces colloques.

Deuxième série d'activités, les ouvrages thématiques publiés en général l'année où il n'y a pas le colloque, donc tous les deux ans aussi. Trois ont été publiés jusqu'à maintenant. Le premier sur Le clergé délinquant, du Moyen-Áge jusqu'à aujourd'bui. Le second sur Les juges, notaires et policiers délinquants, le troisième, l'année dernière, s'intitulait De la déviance à la délinquance. Le prochain devrait porter sur les erreurs judiciaires.

Pour la troisième série, il s'agit des travaux directement réalisés à Dijon, essentiellement par les étudiants que je dirige, donc surtout en histoire moderne, qui portent surtout d'une part sur l'infra-justice, sur les justices seigneuriales, sur l'étude des mentalités et des comportements à partir des archives judiciaires. Évidemment cela ne concerne que la Bourgogne. Il s'agit donc surtout de mémoires de maîtrise, de DEA, de quelques thèses aussi, une cinquantaine de travaux réalisés actuellement. 
Je ne parlerai pas de mes recherches personnelles puisque le thème porte plutôt sur l'équipe que je dirige. Je vous renvoie à mon dernier livre publié il y a quelques mois et qui s'appelle Crimes et Justice aux XVIIème et XVIIIème siècles, chez Imago. Je crois que l'activité du CEH est bien représentative du travail actuel en France à partir des archives judiciaires. Il y a une vingtaine d'années, c'était un travail surtout de type quantitatif. On comptait les crimes, on comptait les sentences, etc. On s'est aperçu qu'il fallait faire cela, mais qu'il y avait pas mal de problèmes et qu'il fallait critiquer.

Dans les années 80 , on s'est dirigé vers des directions nouvelles, mais surtout vers une utilisation des archives judiciaires pour faire autre chose que l'histoire du crime, pour faire une histoire des mentalités, des comportements. Dans les années 90 - et c'est ce que le CEH a essayé d'impulser - on est revenu à une étude plus centrée sur la justice proprement dite, en modifiant le champ des archives étudiées, donc en essayant de descendre aux juridictions de base alors qu'auparavant on s'intéressait surtout aux cours d'appel. En second lieu, on a essayé d'élargir les perspectives, et les différents colloques du $\mathrm{CEH}$ en sont l'illustration, en particulier celui qui a porté sur l'infra-judiciaire.

Ce qui m'amène à parler des perspectives d'avenir, puisque, finalement, c'est toujours le plus intéressant. Je pense que l'histoire de la justice et de la criminalité a beaucoup évolué depuis une vingtaine d'années. Elle était assez nouvelle et les changements ont été considérables dans l'historiographie et dans les méthodes. Je crois qu'il ne faut pas nous endormir sur nos lauriers, qui sont certains, puisque nous avons quand même été des pionniers dans ce domaine.

Mais je crois qu'il faut rebondir sur nos acquis. Dans les années 70, il y avait une sorte d'illusion historiographique qui disait qu'il suffisait de compter les crimes pour voir ce qu'était la criminalité, on s'est aperçu aujourd'hui que cela ne correspondait pas vraiment à grand-chose puisque ce qui est poursuivi est une chose, mais que la réalité délinquante est tout à fait autre chose en général. Je pense que nous sommes en train actuellement, du moins en histoire moderne et en histoire médiévale, peut-être en histoire contemporaine, de retomber dans une 
autre illusion historiographique qui est de dire que tout ce qui ne passe pas devant la justice est traité par l'infra-justice. L'infra-justice est devenue un fourre-tout dans lequel on met tout ce qu'on ne peut pas expliquer ou qu'on explique de façon un peu difficile.

Donc il me semble nécessaire, dans les années à venir, de préciser cette notion d'infra-justice et d'essayer de voir ce qui est traité par la justice, ce qui est traité par l'infra-justice, un concept qu'il faudrait définir de façon plus nette, et ce qui passe par d'autres modes de régulation, ce qu'on pourrait appeler par exemple la para-justice, et enfin tout ce qui n'est pas traité par quoi que ce soit. Il y a des conflits qui ne sont absolument pas réglés et il ne faudrait pas les oublier. Je pense que nous devrions travailler dans cet axe maintenant, mais cela n'exclut pas d'autres directions éventuellement.

\section{Martine Kaluszynski Cerat-IEP de Grenoble}

Il s'agit ici de présenter les laboratoires et structures de recherche qui peuvent accueillir des travaux sur la justice. Avec Laurence Dumoulin nous appartenons au Cerat (Centre de recherche sur l'administration de la ville, le politique et le territoire), laboratoire de science politique rattaché à l'IEP de Grenoble, Fondation des sciences politiques, au CNRS, et à l'université Pierre Mendès-France. Dans ce laboratoire sont travaillées de façon plutôt contemporaines et de manière pluridisciplinaire, toutes les questions ayant trait à l'action publique, l'État, la démocratie mais aussi la question des identités, de la ville, de l'apprentissage institutionnel et du changement politique. Le travail sur les Politiques publiques identifie assez bien la caractéristique de cette structure.

Ce laboratoire n'est pas identifié comme travaillant sur la justice, mais on observe de nombreux travaux qui de près ou de loin touchent à cette question et cela, également, en dehors même de notre seul laboratoire de recherches. Sur le site grenoblois, cette question est traitée à l'Institut d'études politiques par des enseignants-chercheurs intéressés 
par le droit public, d'autres institutions, comme le CEZRSAO (Centre de recherche sur le droit et l'administration publique, dirigé par JeanJacques Gleizal), mènent des travaux sur la police, les politiques pénales, la question de la sécurité, ainsi que le CIDSP (Centre d'informatisation des données en sciences politiques), laboratoire travaillant principalement les questions de sociologie politique, partis, systèmes et comportements politiques, politiques comparées mais où un projet de recherche autour des juges a été élaboré (Kristoff Talin, Anne-Marie Benoit, Crise ou mutation des juges en France? Éléments pour une sociologie des identités professionnelles des magistrats de l'ordre judiciaire). Il y a donc un pôle grenoblois autour de ces questions, traitées dans la pluridisciplinarité et combinant des approches distinctes et complémentaires.

Il y a trois axes qui peuvent se dégager au sein du Cerat, par rapport aux travaux sur la "justice".

Le premier autour de la " justice judiciaire et de travaux menés dans une perspective qui est celle de la sociologie politique du droit ou de la sociologie politique de la justice. Cette approche socio-politique entend travailler le droit et les fonctions de justice comme phénomène propre et révélateur de processus socio-politiques plus généraux. Cette analyse du processus d'élaboration de la norme et des fonctions de justice se retrouve dans les travaux de Jacques Commaille, chercheur associé au Cerat qui participe aux réunions sur l'axe "territoire" et a réalisé un rapport sur cette question (Les enjeux politiques de la territorialisation de la justice. Contributions à une socio-bistoire de la carte judiciaire française publié sous le titre Territoires de justice. Une sociologie politique de la carte judiciaire, Paris, PUF, 2000). Ces travaux vont être élargis à l'Europe. Dans cette filiation, et sous sa direction, des thèses ont été mises en œuvre, sur le personnel ou l'institution judiciaire, sur le Conseil supérieur de la magistrature étudié dans trois pays : France, Allemagne, Italie (AnneLaure Guyot, La régulation des relations entre justice et pouvoir politique : les Conseils supérieurs de la magistrature en France, Italie et Allemagne), sur la police et la justice en Bulgarie (Thierry Delpeuch, Police, justice et pouvoirs dans les pays d'Europe centrale et orientale en mutation démocratique : le cas bulgare, thèse en cours). Un article est également paru sur les usagers de la 
justice (Thierry Delpeuch et Laurence Dumoulin, "La rhétorique de l'usager dans la justice", dans Philippe Warin, Quelle modernisation des services publics? Les usagers au cour des réformes, Paris, La Découverte, 1997, pp. 103-129), et un atelier a été organisé dans le cadre du congrès de l'Association française de science politique (organisé par Laurence Dumoulin et Cesare Mattina au 6ème congrès de l'Association française de Science politique sur les logiques d'action des magistrats).

Le deuxième axe rencontré est fondé autour du pénitentiaire, des prisons, du pénal. Mes propres travaux (voir en particulier, Réformer la société. Les hommes de la Société générale des prisons (1877-1900), Genèses 28, septembre 1997, pp. 75-93), qui dans une dimension socio-historique, s'attachent aux chantiers de réforme sociale dans le domaine juridique et sur la socio-genèse des politiques pénales en France au XIXème et XXème siècles. Jean-Charles Froment a fait une thèse sur les surveillants de prison qui a donné lieu a un ouvrage (La République des surveillants de prison. Ambiguité et paradoxes d'une politique pénitentiaire en France (19581998), Paris, LGDG, 1998). Il travaille également sur la surveillance électronique, sur l'administration pénitentiaire, les questions de sécurité et les politiques pénales. Ensemble, depuis plus d'un an, nous animons le séminaire de troisième année de l'IEP de Grenoble où les étudiants réalisent leur mémoire de fin d'année (équivalent à une maitrise) : Crime(s) et Châtiments : Police(s) et justice(s) XIXème-XXème siècles. Ce séminaire a démarré en 1993 en co-direction avec Jean-Marc Berlière et depuis est renouvelé chaque année sous ma direction et/ou en collaboration, comme ce fut le cas en 1998, 1999 et 2000.

Enfin, le troisième axe correspond à des travaux menés par des enseignants de l'IEP de Grenoble autour de la justice constitutionnelle (Jean Marcou, Justice constitutionnelle et systèmes politiques. États-Unis, Europe, France, PUG, 1997) et du juge administratif (Henri Oberdoff et Guy Gardavaud (dir.), Le juge administratif à l'aube du XXIème siècle, PUG, 1995). 


\section{Laurence Dumoulin}

Cerat-IEP de Grenoble

Outre ces travaux portant spécifiquement sur la justice, sont développées un certain nombre de recherches qui, de façon plus indirecte, ont à voir avec la justice, soit parce quelles sont mises en œuvre à partir de matériaux juridiques ou judiciaires, soit parce quelles ont trait à des thèmes connexes comme celui de la sécurité.

C'est une des particularités de la socio-histoire du politique (Martine Kaluszynski et Sophie Wahnich, L'État contre la politique, les expressions historiques de l'étatisation, Paris, L'Harmattan, coll. "logiques politiques", 1998) que de se pencher sur les processus d'élaboration normative et de construction des catégories juridiques. Dans les thèses d'Aurélie Billebault (La diffamation politique en France : histoire et sociologie de l'apprentissage d'une règle de civilité, XIXème-XXème siècle, thèse en cours) et de Nathalie Dompnier (La règle du jeu. La codification de la "sincérité" des opérations électorales lors des scrutins législatifs en France depuis 1848, thèse en cours), sont utilisées comme matériaux des revues juridiques, des archives judiciaires qui permettent de travailler sur la manière dont sont juridiquement construites et judiciairement mises en œuvre les notions de diffamation politique et de fraude électorale.

Dans ses travaux sur la société générale des prisons ("Les artisans de la loi. Espaces juridico-politiques en France sous la IIIème République", Droit et société, 40, 1998, pp. 532-562, ainsi que "La revue pénitentiaire 1877-1900. Un réseau de réformateurs sociaux”, dans Frédéric Chauvaud et Jacques-Guy Petit (dir.), L'histoire contemporaine et les usages des archives judiciaires (1800-1939), Paris/Genève, H. Champion/Slatkine, 1998, pp. 269-280), Martine Kaluszynski utilise comme premier matériau la revue juridique produite par ce laboratoire de la législation pénale.

Dans une approche de sociologie politique plus classique, quelques travaux utilisent également de façon privilégiée les sources judiciaires. Cesare Mattina, par exemple, tente de comprendre le fonctionnement du clientélisme politique et du vote d'échange à partir d'entretiens avec les 
acteurs concernés mais aussi d'affaires jugées pour ce motif (La gestion $d u$ pouvoir local et les réseaux de clientèle à Naples et à Marseille dans les années 1980, thèse en cours). L'analyse des dossiers judiciaires permet alors de reconstituer les mécanismes concrets du phénomène ainsi que de comprendre les différentes logiques (judiciaire, politique, sociale) à l'œuvre.

Véronique Pujas développe une approche semblable dans une thèse récemment soutenue (Les scandales politiques en France, en Italie et en Espagne : constructions, usages et conflits de légitimité, Institut universitaire européen, Florence, janvier 1999), consacrée aux scandales politiques, au sein de laquelle la magistrature est largement approchée, dans ses liens avec le politique, et notamment à partir d'entretiens. Le judiciaire est ici envisagé comme une scène entretenant des interactions multiples avec des scènes parallèles, plus ou moins concurrentes, en l'occurrence la scène médiatique et la scène politique.

En ce qui concerne les travaux qui traitent de problématiques non directement axées sur la justice et le droit mais les intéressant voire les recoupant largement, on peut rappeler que le Cerat a une grande tradition de travail sur l'action et les politiques publiques. Il pose à cette occasion des questions de responsabilité : de l'État vis-à-vis de ses administrés et de l'administration à l'égard de ses usagers. Dans les recherches menées par Bruno Jobert ("Les nouveaux usages du droit dans la régulation politique" (2000), par Philippe Warin ("La performance publique : attente des usages et réponses de ministères", Revue politique de management public, vol. 17, n² , juin 1999, pp. 147-163) sont évoquées à propos de recompositions actuelles de l'État, les repositionnements de la justice dans le dispositif de régulation sociale et politique.

Les problématiques de la juridicisation et de la judiciarisation du politique sont ainsi explorées, notamment dans un rapport de recherche en cours ainsi que dans un ouvrage collectif récent (Jacques Commaille, Laurence Dumoulin et Cécile Robert (dir.), La juridicisation du politique. Leçons scientifiques, op. cit.). Le droit est envisagé comme nouveau mode de régulation ou plus exactement comme un mode de régulation investi de façon renouvelée.

Parallèlement, les thématiques de la sécurité et de l'insécurité don- 
nent également lieu à de multiples recherches que ce soit dans le cadre du Cerdap avec Jean-Jacques Gleizal, Jean-Charles Froment (dir.), Les mécanismes de régulation sociale. La justice, l'administration, la police, Montréal/Lyon, Éd. du Boréal/PUL, 1998 et Les politiques locales de sécurité, Voiron, Éd. La lettre du cadre territorial, 1998) ou au sein du Cerat avec Sébastien Roche (Le sentiment d'insécurité, Paris, PUF, coll. "Sociologie d'aujourd'hui", 1993 et La société incivile. Qu'est-ce que l'insécurité ? Paris, Seuil, coll. "L'épreuve des faits ", 1996). Est assez proche de ces thèmes le séminaire "Crimes et châtiments police et justice XIXème-XXème siècles" en place depuis un peu plus de cinq ans à partir de contenus et de collaborations variables, et destiné aux étudiants de troisième année de IEP. Lieu de formation à la recherche, c'est aussi un lieu de production puisqu'une cinquantaine de mémoires a d'ores et déjà été réalisée dans ce cadre.

On ne saurait conclure ce bref panorama des productions grenobloises en matière de justice sans évoquer les projets en cours. Trois entreprises se profilent qui, là encore, donneront lieu à des échanges et collaborations : une étude sur le non-recours aux institutions et services étatiques, en particulier la Justice et la Police ("Le non-recours aux services de l'État. Mesure et analyse d'un phénomène méconnu”, recherche sous la dir. de Philippe Warin) ; une enquête sur le processus législatif d'adoption d'une innovation technologique porteuse d'effets sur le système répressif, l'assignation à domicile sous surveillance électronique (rapidement désignée bracelet électronique ("Sécurité et nouvelles technologies...", recherche sous la dir. de Jean-Charles Froment et Martine Kaluszynski) ; enfin un séminaire "sécurité et société" (organisé par Jean-Charles Froment et Sébastien Roche) qui fédérera les différents partenaires évoqués : CIDSP, Cerat, Cerdab, IEP et rassemblera des énergies, dispersées sur le site, mais que des intérêts convergeant pour les questions de régulation des sociétés actuelles réunissent. 


\section{Michel Cassan}

Historien, université de Limoges

Je parlerai, dans le cadre de cette session- des aspects un peu méthodologiques, un peu historiographiques aussi, d'un thème qui nous déporte dans le temps, puisqu'il s'agit des XVIème-XVIIIème siècles, Révolution exclue et d'un groupe social ou professionnel, d'abord professionnel, qui a été qualifié, il y a maintenant une douzaine d'années par Jean Nagle, d'officiers "moyens".

L'officier "moyen" est principalement membre d'un présidial, d'une sénéchaussée, d'une élection, d'un grenier à sel. Et on pourrait discuter assez longuement sur cette définition de l'officier "moyen", qui est empirique, puisque l'officier "moyen" est d'abord celui qui ne possède pas une charge anoblissante. Et le seuil inférieur c'est la recherche qui pourra le déterminer. On sait que ces officiers "moyens" sont nombreux dans la France de l'Ancien régime, puisque ce sont les gros bataillons d'un État, d'un corps appelé quatrième État par Charles Loyseau au début du XVIIème siècle, qui passe de 4.000 membres sous le règne de François Ier à environ 46.000 sous le règne de Louis XIV. Et probablement que $90 \%$ des membres de ce quatrième État sont des officiers "moyens".

Très rapidement, rappelons les positions et les enjeux historiographiques. Depuis la fin du XIXème siècle, il y a eu quatre moments dans cette historiographie. Le premier, ce sont les années 1880/85 à 1930, avec une série de monographies de cours de justice réalisées par des magistrats érudits. C'est la posture du magistrat lettré du XVIIIème siècle, voire du XVIIème siècle. Ce sont des hommes amenés à faire des discours de rentrée, qui ont été étudiés par Jean-Claude Farcy. Certains prolongent ces discours de rentrée et donnent des monographies qui sont des histoires de la compagnie dans laquelle ils siègent. On peut remarquer que, globalement, ils reprennent quelques thématiques. La thématique du déclin du tribunal au XVIIIème siècle, qui était d'ailleurs déjà présente peu avant la Révolution et ils raisonnent, quand ils veu- 
lent présenter des thèmes, celui de la commune. On est là tout à fait en rapport avec des débats de la fin de la Troisième République.

Le second moment, celui que je pourrais appeler le moment Roland Mounier et ses élèves, 1945-1980, avec une grande thèse sur la vénalité des offices sous Henri IV et Louis XIII, et une série d'études monographiques. Ce sont des thèses d'État, thèses d'histoire institutionnelle et sociale, qui privilégient le XVIIIème siècle et les cours souveraines et que vous avez dans toutes les bibliothèques.

Troisième moment, les années 1985-1995, la vaste enquête sur la genèse de l'État moderne, menée à l'échelle européenne, dans le cadre d'une action programmée. On trouve là une démarche comparatiste, une méthode, la prosopographie, avec ses apports et ses limites, des résultats, un approfondissement des connaissances des institutions, un déchiffrement des systèmes administratifs, des liens familiaux, de l'héritage et de l'alliance matrimoniale dans la circulation des charges, la place des réseaux de sociabilité professionnelle, des liens clientélaires et l'extension de la vénalité à des offices municipaux et notamment parisiens. Très rapidement, les limites de ces analyses. La prosopographie - et c'est son intérêt - ausculte l'individu sous toutes ses faces, origines sociales, géographiques, cursus scolaire, modalités d'entrée dans l'office, durée d'exercice de la charge. Ces données sont traduites en chiffres et en moyennes. Résultat, on aboutit à un individu-type qui est censé être représentatif. C'est évidemment intéressant statistiquement. Cela peut être une abstraction comptable. Il faut avoir ces données, mais il ne faut pas les sacraliser et il faut essayer de les dépasser. D’où un problème très net : comment les dépasser? C’est un peu la recherche actuelle. L'autre écueil de la prosopographie, c'est la catégorisation ou la classification sociale. Trop souvent encore, le découpage se fait à l'aide de grilles qui sont assez proches de notre société contemporaine, même si elles sont revisitées, même s'il y a des tentatives pour les affiner. La société de l'Ancien Régime est évidemment très différente. Là on a des limites qu'il faut avoir présent à l'esprit pour éviter les risques d'anachronisme.

Le quatrième moment est le moment contemporain. C'est vraiment l'actualité. Depuis 1995, tout cadre global a disparu, l'action program- 
matique a disparu. Il y a un émiettement de la recherche, quelques points d'ancrage institutionnel, des questionnements qui sont plus mouvants. Par commodité, il y en aurait deux à souligner.

D'abord le territoire. Quel degré d'analyse ? La monographie, c'est-àdire une cour, la province, le pays, pour le chantier européen, puisque la vénalité est un phénomène mondial, ce qui avait été dit par Mounier et par Goubert, il y a déjà plus de quarante ans.

Deuxièmement, c'est la thématique. Quelle lecture privilégier ? Une lecture juridique ? Une lecture culturelle ? Ou un approfondissement des chantiers sociaux, de la dimension sociale du sujet ? Ou essayer de lier le tout ? Enfin, la priorité doit-elle être accordée à l'office ou à l'officier? Ce qui modifie évidemment les angles d'approche.

Deuxième partie, les perspectives de recherche, appliquées au monde des officiers "moyens", des perspectives de recherche que je mets en débat. Premièrement, on peut rêver à des études macroscopiques et provinciales descriptives afin de déterminer l'effectif des officiers "moyens". Le maillage administratif d'une contrée, le poids de l'État en distinguant le nombre des offices et le nombre des officiers, ce qui n'est pas identique puisque vous avez des phénomènes de cumul. Il faudrait là une enquête de longue durée de façon à prendre la mesure de la place de l'État d'offices et de le confronter à l'État des offices seigneuriaux. Il faut bien opérer une distinction entre les môles de présence, voire de résistance seigneuriale à l'État administratif royal.

Deuxième thème, la dynamique sociale des administrateurs de rang moyen, puisque l'accès à l'office est une des voies, assez rare, de mobilité sociale ascendante, individuelle qui est en réalité précédée et accompagnée d'un investissement familial. Là encore il faudrait arriver à des modélisations, possibles à condition de tenir ensemble plusieurs paramètres. Un, le marché de l'office. Deux, le marché matrimonial. Trois, le statut des intéressés, cadets ou aînés. Quatre, le système des coutumes et des règles successorales en usage. Il faut vraiment avoir les quatre éléments, les quatre données pour prétendre arriver à des résultats fiables. Il faut, en contrepoint, se défier de la notion de stratégie personnelle 
ou de stratégie matrimoniale et familiale. En tout cas, il faut éviter d'en systématiser l'emploi. C'est trop souvent, dans l'esprit des historiens, une reconstruction qui minimise ou évacue toute dimension aléatoire. Or, le marché de l'office est un marché spéculatif. Et, comme tout marché spéculatif, il est soumis à des crises.

Le marché de l'office serait le troisième thème. Il est très mal connu. On arrive à connaître les modalités de transmission de l'office et les grandes lignes du cours de l'office. Ce que l'on sait sur le premier point, c'est qu'il y a une distinction entre l'acquisition, la transmission familiale, de père à fils ou de beau-père à gendre. Mais il y a vraisemblablement les différences de chronologie. Cela a un retentissement sur la dynamique sociale.

Sur le second point, le cours de l'office, les grandes lignes sont saisissables. On sait qu'il y a eu une augmentation, voire une flambée de la valeur des offices sous les règnes d'Henri IV et de Louis XIII, une déflation rapide et brutale, et durable sous Colbert, une stagnation probable dans la seconde moitié du XVIIIème siècle, si l'on se réfere aux plaintes des détenteurs. Simplement là c'est une appréciation très impressionniste. Il faut passer à des faits dans une démarche néo-positiviste. Et il faut s'appuyer sur des traités d'office, que l'on trouve dans les minutiers. Ce sont des documents rares, mais qui donnent une foule de renseignements.

Il faudrait, à l'aide de ces traités d'office - et cela a commencé - vérifier les hypothèses suivantes et ce ne sont que des hypothèses. L'office est-il une affaire plus familiale que personnelle, une fonction qui reste dans la lignée plus qu'elle n'entre dans la communauté des biens d'un ménage. Est-ce une fonction achetée à crédit plus qu'au comptant ? Est-ce un bien qui n'est pas jugé suffisant par un créancier comme gage d'hypothèque, ce qui serait bien la preuve de la dimension spéculative de l'office ?

Autre thème qu'il faudrait aborder, celui de l'administrateur au travail et ses contreparties matérielles ou symboliques. Ce que l'on sait est très succinct. Vous pourrez ainsi voir combien il faut encore travailler. Nous connaissons les calendriers des tribunaux, les règlements, les conditions d'accès aux corps par la pratique de l'examen. En revanche il y a beau- 
(1) Table ronde dont les actes ont été publiés dans les Cabiers du centre de recherches historiques de l'EHESS en octobre 1999. coup d'interrogations. On ne sait à peu près rien des rémunérations directes ou indirectes des administrateurs. On connaît mal la culture juridique des magistrats, leur degré de technicité et de professionnalisation, qu'il faudrait là rattacher à une hypothèse centrale. L'État a-t-il recherché un personnel compétent et qualifié pour atteindre ses objectifs administratifs et politiques?

Sur le travail du magistrat, j'insisterai simplement sur un point. On ne peut pas envisager le travail du magistrat de manière isolée. C'est ce que nous avons essayé de faire l'année dernière avec cette table ronde dont j'ai parlé. ${ }^{(1)}$ Nous avons essayé de réfléchir au travail de l'institution sur elle-même. Il apparaît bien que le travail du magistrat, envisagé de manière isolée, ne peut pas être traité. L'officier n'a pas une pratique singulière. L'officier magistrat est d'abord membre d'une compagnie qui lui dicte, lui impose, lui suggère sa loi.

Le magistrat exerce des compétences, mais il les apprend aussi dans le cadre d'une institution qui a sa pratique propre, sa dynamique ou sa routine. Il y a là tout un jeu à décrypter, toute une analyse à faire. On ne peut saisir cette analyse que lors des conflits entre les membres du tribunal. C'est l'occasion d'un dévoilement des modes de travail et de fonctionnement. Et l'opportunité de mesurer l'écart entre la norme, la pratique et le retour à une autre norme couchée dans un nouveau règlement.

Deuxième point, l'institution produit un certain volume de travail et qui justifie son existence. Cela suffit-il à justifier l'existence de ses membres ? Si le travail vient à manquer, quels sorts sont réservés à l'institution et à ses membres ? La destinée de l'institution et la destinée des membres de l'institution ne sont pas nécessairement identiques, parallèles, bien au contraire. Si on posait la question différemment, on pourrait se demander si les officiers, lorsqu'ils achètent une charge, achètent une fonction, un statut, ou les deux et quel est le lien. J'avais des hypothèses mais ce serait trop long de les exprimer.

Dernier axe qui devrait retenir notre attention me semble-t-il, ce sont les activités extra-professionnelles des administrateurs de la justice, la frontière entre sphère professionnelle et sphère publique qui est souvent poreuse. J'ai évoqué au début de ce court exposé, les discours de rentrée 
des magistrats qui ont été étudiés au XIXème siècle, qui existaient bien sûr au XVIIIème siècle, où l'on voit l'investissement et le réemploi, dans les discours, de lectures faites durant le temps du loisir.

Autre activité professionnelle plus classique, la participation des officiers à la gestion des villes, comme marque d'insertion locale, avec tous les débats entre les gens du roi et les gens de la ville. De la même manière, on peut saisir les magistrats ou les administrateurs dans leur for privé, à travers leurs pratiques savantes, à travers leur participation à des réseaux : réseaux académiques, réseaux de sociétés agricoles, réseaux de franc-maçonnerie. Il y a là un chantier qui reste très ouvert, sans oublier qu'au XVIIème siècle, les magistrats ont été vraisemblablement l'aile marchante de la Contre-Réforme ou de la Réforme catholique. Il y a un basculement d'une élite catholique militante au XVIIIème siècle à ses successeurs de la fin du XVIIIème siècle, attirés par la spéculation maçonnique.

En conclusion, cette étude des officiers "moyens", qui a débuté par quelques publications depuis maintenant 1996 (Les officiers "moyens" à l'époque moderne. France, Angleterre, Espagne. Études réunies par Michel Cassan, Limoges, Pulim, 1998), s'inscrit maintenant dans l'histoire sociale et culturelle des institutions. Elle privilégie les stratégies d'investissement des institutions, sans céder à une démarche de la conspiration qui verrait dans le tribunal, un lieu à instrumentaliser pour obtenir le - ou du pouvoir. Je crois qu'elle doit être attentive aux différences provinciales, aux oppositions entre façade maritime et province de l'intérieur, au moins autant qu'à l'articulation entre centre et périphérie, centralité parisienne et marges du royaume.

Et elle doit placer au rang des priorités la culture et l'histoire matérielle de l'institution, que ce soit ses édifices, essayer de cerner Comment pensent les institutions. C'est le titre du livre de Mary Douglas (Paris, La Découverte/M. AU.S.S. 1999) auquel je fais référence. Comment elles font penser leurs membres ? Comment leurs membres les font vivre ou les abandonnent, puisqu'au fond, dans la longue durée, l'histoire des sièges présidiaux semble l'histoire d'un déclin au XVIIIème siècle ? Un déclin dont on n'a même pas dressé de façon solide, la validité et la réalité. 
Michel Porret. Pour aller à l'essentiel, dans le cadre d'une approche hors de l'espace judiciaire français, j'aimerais souligner, d'une certaine manière, quelque chose qui est ressorti de la communication de JeanClaude Farcy, mais d'une façon un peu sous-jacente et non-dite. Je crois que nous sommes encore beaucoup trop marqués par une historiographie qui pense la modernité judiciaire et pénale, uniquement à partir du modèle de rupture qui a mis fin à l'Ancien Régime en France, en oubliant quelquefois qu'il y a des modèles périphériques européens qui ont construit une modernité judiciaire.

Marc Bloch et Lucien Fèbvre détestaient le mot de "précurseurs" mais on peut l'utiliser très rapidement à des fins pédagogiques. Je crois que se sont construites des modernités judiciaires et pénales avant la fin de l'Ancien Régime, tel qu'il est donné en 1789 pour l'histoire nationale française.

Les quelques remarques que je vais faire sur l'Histoire de la justice désignent des travaux et des recherches qui ont lieu dans le cadre du département d'histoire générale de l'Université à Genève, où j'enseigne l'histoire moderne largement, avec un important dossier consacré aux Lumières que je fais commencer vers 1650 et que je fais terminer, en gros, à la fin du mouvement idéologue, vers 1820-1830. Contrairement à tous mes collègues qui ont parlé avant moi, je ne suis pas dans une équipe. Je parle principalement en fonction d'une recherche, sur laquelle je fais travailler mes étudiants.

Il y a un thème central dans ces recherches, dont je voudrais vous faire part en quelques minutes. C'est le problème qui m'a intéressé dans un livre que j'ai écrit et publié en 1995, lequel essayait de comprendre comment, sous un régime arbitraire, c'est-à-dire sans cadre légal, fonctionnait dans la pratique ce que l'on peut appeler la qualification du crime et la motivation de la peine. J'ai essayé de comprendre comment les usages judiciaires et les pratiques pénales, entre 1670 et la fin du XVIIIème siècle, autour de la qualification et de la motivation, comment l'arbitraire d'une façon extrêmement cohérente, construisait ses propres normes de proto-légalité qui allait mettre l'arbitraire dans l'impasse. 
La recherche à Genève sur l'Histoire de la justice, peut être le privilège des petites villes-État qui ont inventé au XVIème siècle la République, de type aristo-démocratique. Cette recherche se base sur un matériau d'archives exceptionnelles à peu près ininterrompues du XVIème siècle à la fin de l'Ancien Régime. A Genève, la fin de l'Ancien Régime se situe en 1794. Une période politiquement trouble va jusqu'en 1798 où nous sommes intégrés dans l'espace français puisque Genève devint chef-lieu du département du Léman jusqu'en 1813. Comme l'a écrit Michelet, arrive à ce moment-là deux instruments de la modernité judiciaire et pénale : le code pénal et la guillotine. Après c'est une autre histoire qui s'ouvre avec le XIXème siècle.

Il y a donc un matériau d'archives exceptionnel qui permet de travailler sur des usages sociaux, sur des pratiques pénales, d'une manière ininterrompue entre le XVIème et le début du XIXème siècle. C'est un travail sur lequel j'essaie de mélanger à la fois des textes tirés des archives judiciaires, des textes de la doctrine et des textes des sources de la loi, que ce soit des textes genevois et républicains, ou des textes plus propres à la culture juridique européenne.

Autour de cette histoire de la qualification et de la motivation sous l'Ancien régime, s'est ajouté depuis trois ou quatre ans un chantier sur lequel je travaille et fais travailler plusieurs étudiants, c'est l'histoire d'un nouveau champ, qui va devenir d'ici quelques années un objet sur lequel beaucoup de travaux seront faits, celui qu'on appelait la médecine judiciaire à partir de Zachias. Et c'est celui qu'on va commencer d'appeler en France à partir de 1770 la médecine légale, puisque c'est un néologisme qui apparaît, vous le savez, dans le supplément de l'Encyclopédie Diderot-d'Alembert.

Toujours le même problème sur les archives judiciaires, essayer de souder ce qui lie les pratiques et ce qui lie les usages judiciaires et pénaux dans un contexte à la fois institutionnel, intellectuel et doctrinal. Je le répète, ce qui m'intéresse c'est de comprendre comment fonctionne la justice traditionnelle, sans supposer qu'elle sera condamnée à évoluer vers le Code pénal de 1791. 
C'est ce que j'essaie de faire avec mes étudiants, comprendre la logique, au moment où j'étudie les dossiers, dans le contexte de l'Ancien Régime, dans le contexte des années 1720-1730 et de ne pas projeter la fin de cette justice d'Ancien Régime en fonction de ce qui va la condamner, c'est-à-dire le régime de la légalité, des délits et des peines.

L'objectif de ces recherches, pour venir au centre de ces quelques remarques, c'est d'essayer de mettre en lumière un modèle, qui est encore un peu dans l'enfance, un modèle qui montrerait qu'il y a une justice particulière, inséparable d'une culture politique, d'une culture juridique et d'une culture institutionnelle, d'un système politique qui est une république. On ne peut pas penser cette modernité si on n'a pas en tête qu'une justice ne peut être comprise que dans son cadre politique.

$\mathrm{Si}$ on regarde et si on reprend certaines phrases, par exemple chez Montesquieu, qui associe la douceur des mœurs au système républicain, d'une justice adaptée aux mœurs, c'est extrêmement intéressant de voir qu'il y a des caractéristiques dans l'institution pénale genevoise, à partir de la fin du XVIIème siècle, qui montrent que se mettent en place déjà certains éléments d'une modernité institutionnelle plus tardive.

Quelques exemples.

L'abolition légale de la question en 1738, près la Suède. Un usage croissant de la prison domestique à partir du début du XVIIème siècle. A Genève, on va très systématiquement essayer de remplacer le bannissement par des formes anciennes d'incarcération, soit à l'intérieur du logis privé de l'individu, ce qu'on appelle la prison domestique. On peut être condamné à quinze ans de prison domestique à Genève, c'est-à-dire pendant quinze ans, on va devoir simplement travailler et rentrer dans son logis le soir, sous un contrôle fait par des magistrats de justice. $\mathrm{Ou}$ encore quelque chose qui entre en crise à partir de 1750, le recours extrêmement prudent à la peine capitale, qui disparaît presque des pratiques à partir des années 1750 .

Il y a d'autres éléments qui montrent que finalement l'institution pénale genevoise républicaine entre dans une sorte de proto-utilitarisme, basé sur le recours de plus en plus croissant, depuis les années 17201730, à une culture de l'expertise, c'est-à-dire à deux types d'expertises, 
essentiellement des expertises de type médico-légal et des expertises, techniques notamment, dans les affaires d'effraction, de vol nocturne, de vol en association, etc.

Il y a véritablement une sorte de rationalité, peut-être "protestante", qui marque les pratiques pénales et les usages judiciaires à Genève, durant l'Ancien Régime, avec un mot - utilisé avec prudence notamment par les historiens de la médecine et des sciences - une obsession de la certitude, de l'incrimination. A Genève, durant tout l'Ancien Régime, les procureurs généraux ne cessent de proclamer : "Une bonne justice avec une certitude des incriminations, finalement est une justice qui montre que le gouvernement est juste et équitable.”

Quelques gros dossiers sont en cours chez des étudiants. Je ne vais pas mettre en avant certains travaux que je mène d'une manière parallèle aux mémoires de DEA, aux mémoires de licence, à certaines thèses qui commencent. D'abord une importante thèse est en cours de rédaction, son objet semble assez inédit dans l'historiographie européenne. Après un très bon mémoire, dont une version va paraître dans le prochain numéro de Crime, Histoire et Société, une de mes étudiantes étudie très systématiquement la mise en place de la défense des criminels. A Genève, depuis 1738, la défense par des avocats des criminels de droit commun est légalisée. Elle va étudier, entre 1738 et 1798, cette pratique. Il y a un corpus extraordinaire de plaidoyers, de requêtes d'avocats qui montrent simplement que, dans un système républicain, l'importance de l'individu prend une forme exceptionnelle devant les tribunaux à partir du début du XVIIIème siècle, que ces individus soient des petits voleurs de bas étage ou que ce soient des individus ayant une surface sociale plus large. Je crois que se joue dans l'institution genevoise, très tôt au début du XVIIIème siècle, quelque chose qui montre où, dans la longue durée, va s'ancrer dans la culture juridique des Lumières, l'État de droit.

Il y aussi certains contentieux criminels que je fais étudier par mes étudiants. En voici quelques exemples. Un premier mémoire sur les prostituées entre 1720 et 1790 qui a montré à quel point les "libertines" faisaient précocement l'expérience historique de l'incarcération ; les 
bandits associés, des travaux maintenant sur les récidivistes ou sur les soldats criminels. Voilà quelques thèmes étudiés pour l'instant dans certains mémoires de licence.

Et puis il y a un autre important dossier. Frédéric Chauvaud en dira un mot tout à l'heure puisque c'est aussi un des champs sur lesquels il travaille avec beaucoup de nouveautés. C'est le dossier de l'expertise. Ce qui m'a frappé dans le corpus judiciaire genevois du XVIIIème siècle, c'est que l'on peut faire une histoire d'une triple expertise qui est parfaitement cohérente dans sa mise en place institutionnelle. C'est curieux de voir à quel point les animaux, les individus et les choses deviennent objet d'expertise dans l'institution genevoise au XVIIIème siècle.

L'un de mes étudiants a publié un livre, il y a deux ans, qui a montré comment se construit à partir du début du XVIIIème siècle, ce que l'on pourrait appeler l'expertise zoologico-légale, qui devient très importante, puisque l'appareil de justice est sur le front des épizooties durant tout l'Ancien Régime et lorsqu'il faut décider d'abattre un troupeau - ce qui est un traumatisme extrêmement profond pour un paysan - on ne peut pas abattre ce troupeau en l'absence d'expertises, qui montrent la pathologie des troupeaux devant être abattu pour des raisons de police du bétail.

L'autre champ expertal est celui des expertises médico-légales autour de la blessure, du corps blessé, de l'homicide, des crimes sexuels, des suicides. Je vous donnerai simplement un chiffre. Au début du XVIIIème siècle à Genève, le corpus des documents judiciaires qui sont médicalisés atteint 7 ou $8 \%$. A la fin du XVIIIème siècle, on frise 30 à $35 \%$. Il y a donc une multiplication de quatre ou cinq sur l'ensemble du contentieux criminel.

Folie, crimes de sang, crimes sexuels : le médico-légal dessine un champ immense. Par exemple, l'émergence de nouvelles exigences socio-professionnelles entre les chirurgiens et les médecins, la blessure extérieure, le diagnostic intérieur pour empoisonnement, ou encore ce qui est peutêtre encore plus intéressant et plus neuf, les expertises médico-légales qui permettent à la Justice de se prononcer très précocement sur l'intention criminelle de celui qui frappe. La profondeur et le nombre des blessures, à partir de 1739-1740, deviennent des éléments qui permettent de cons- 
truire un discours juridique sur la dangerosité - entre guillemets puisque c'est un mot du XIXème siècle - de celui qui a frappé.

Dernier chantier en cours et sur lequel je travaille aussi dans le cadre de l'expertise. Je vous ai dit qu'il y avait trois éléments : les animaux, les humains, les objets. D’une manière extrêmement cohérente, parallèle, l'expertise typographico-légale, devient d'un usage croissant à partir des années 1730-1740. Et à Genève, tout acte de censure politique contre les mauvais livres, essentiellement les livres politiques, ne peuvent pas fonctionner si ces livres n'ont pas été soumis, d'une manière très minutieuse, à une expertise typographico-légale qui permet notamment de reconstituer les réseaux, la fabrication, etc.

Deux mots encore sur le beau travail qui vient de paraître dans Crime, Histoire, Société, une grande enquête de l'un de mes étudiants qui a travaillé sur la manière dont les réseaux criminels, dans la région alémaniques, fonctionnent ("la criminalité associative") à partir de 1660-1670 et combien jusqu'à la fin du XVIIIème siècle, cette criminalité associative, qui cumule toute une série de circonstances aggravantes légitime quelque chose dont on a très peu d'informations dans l'historiographie, la coopération policière durant l'Ancien Régime entre des juridictions et des Etats qui n'ont pas les mêmes systèmes religieux, qui n'ont pas les mêmes systèmes de juridictions. A Genève, une étudiante a fait son mémoire là-dessus. On a une énorme documentation intitulée Avis de Recherche. Arrivent à Genève des centaines d'avis de recherche, manuscrits ou imprimés, qui viennent des juridictions qui enserrent la République jusqu'à la fin du XVIIIème siècle. De Genève partent aussi des avis de recherche et on peut travailler sur ces documents et montrer comment se construit une sorte d'anthropologie empirique avant la date où tous les éléments de la description physique, culminent avec cette obsession de la justice d'Ancien régime. C'est-à-dire qu'il y a un lien entre la difformité morale et le comportement criminel.

Voilà donc quelques éléments très rapidement dits autour de ce dossier de recherche ouvert à Genève, simplement autour de mon enseignement qui consacre une partie à l'Histoire de la justice pénale, mais dans 
une réflexion générale de type Lumières/Ancien Régime, avec un élément sur lequel je terminerai. Je crois qu'il est très important maintenant - nous avons parlé de Foucault aujourd'hui - dans les travaux sur la justice, d'abandonner le paradigme dominant de Surveiller et punir, qui était de dire : "On peut penser la justice à partir d'un paradigme d'intentionnalité punitive.” Il ne s'agit pas ici de réhabiliter la justice d’Ancien Régime, mais de comprendre une justice d'Ancien Régime qui, d'une certaine manière - peut-être d'une façon très forte dans un système politique comme Genève - avait des comptes à rendre aux justiciables-mêmes qu'on envoyait sur l'échafaud ou dans l'exil du bannissement.

\section{Jean-Marie Augustin}

Historien du droit, Institut d'bistoire du droit, université de Poitiers

Les recherches effectuées dans le cadre de l'Institut d'histoire du droit de Poitiers sont un peu dispersées, en raison même de la personnalité de chaque chercheur. Vous évoquiez l'histoire du Parquet, Madame Christine Mengès-Le Pape a travaillé sur le "statut des gens du roi à la fin de l'Ancien Régime" qui est paru dans l'ouvrage collectif dirigé par Monsieur Jean-Marie Carbasse, consacré au sujet. Elle participe aussi au Centre d'étude d'histoire Juridique (équipe de recherche de l'université de Paris II associée aux Archives nationales et au CNRS). Sous la direction de Madame Le Pape, Monsieur Christophe Lossot prépare sa thèse sur "La justice d'Ancien Régime vue par les juristes et les publicistes de l'Empire : mythes et réalités".

Une autre recherche est menée sous la direction de Monsieur François Saint-Bonnet sur les rapports entre justice et politique, ainsi que sur la juridiction administrative au XIXème siècle.

Il faut encore rappeler les thèses de Monsieur Didier Veillon sur Les magistrats dans le ressort de la cour d'appel de Poitiers, soutenue en décembre 1996, qui doit faire l'objet prochainement d'une publication, et de Monsieur Éric Dumontet sur La maîtrise des eanx-et-forêts de Poitiers de la réformation à la Révolution (1661-1790). Des mémoires de DEA ont été 
également consacrés aux justices de paix, notamment à la Flotte-en-Ré (Charente-maritime) sous la Révolution et à Saint-Georges-lesBaillargeaux (Vienne) sous la Second Empire. Une recherche pourrait être approfondie vers cette justice de proximité, des justices seigneuriales de l'Ancien Régime aux justices de paix. Jusqu'ici elle a été plutôt menée en milieu rural, mais elle pourrait aussi être faite des juridictions municipales aux justices de paix dans les cantons urbains.

\section{Frédéric Chauvaud}

Professeur d'histoire contemporaine, responsable de l'équipe "Conflictuosité" $d u$ Gerbico

L'intérêt pour la justice, sujet d'enquête délaissée ou tout simplement ignorée, en dehors des seuls spécialistes d'histoire du droit, s'est métamorphosé. La justice est en effet devenue un objet de recherche "accrédité" par l'ensemble de la communauté des chercheurs, même si les travaux réalisés, hormis ceux qui traitent de la justice pénale, restent peu nombreux. Deux indices l'attestent. L'engouement récent pour la justice et la légitimité des approches qui ne sont pas strictement juridiques se sont manifestés lors du XIXème Congrès international des sciences historiques, qui s'est tenu à Oslo au milieu du mois d'août 2000. Cette importante manifestation scientifique avait retenu, dans le cadre de l'une de ses sessions et pour la première fois, le thème de la justice. Des historiens français venant des sciences humaines y ont apporté une contribution importante. Par sa seule existence, cette semaine de débats a bien constitué une sorte de "point tournant" en conférant aux recherches sur la justice une "visibilité" internationale. Le second indice se rapporte aux choix éditoriaux de la revue Société et Représentations. L'une des toutes prochaines livraisons de ce périodique aura pour thème la justice. S'il existe des revues de grandes qualité comme Histoire de la Justice, Droit \& Société et quelques autres, dont la raison d'être et la visée sont de cerner le fonctionnement de l'institution et de la société judiciaires, tout en ne négligeant pas les évolutions et les lectures du Droit, jamais une revue de 
sciences humaines n'avait encore consacré un numéro entier à la justice. Ces deux indices semblent indiquer que l'intérêt pour la Justice ne relève ni d'un frémissement passager ni d'un bruissement fugace.

Dans cette perspective l'équipe "Conflictuosité" du Gerhico a utilisé les archives judiciaires, de l'époque moderne et contemporaine, comme une porte ouverte sur la vie quotidienne, puisque peuvent être saisis l'existence des domestiques, les cultures populaires, les figures du mépris et de la haine... Il s'avère en effet possible de restituer les comportements, de définir selon les perceptions des contemporains les catégories sociales, d'identifier les repères mentaux et la hiérarchie des intérêts des hommes et des femmes du passé. Mais l'équipe "Conflictuosité" s'intéresse à l'histoire de la justice en temps que telle. Dans l'immédiat, la réflexion a plutôt porté sur le repérage des champs à investir.

Six directions différentes ont été retenues. La première se rapporte à l'espace. La notion de territoire de justice est au centre de cette approche. Il ne s'agit pas seulement des éléments constitutifs de l'histoire de la carte judiciaire, avec ses bornes administratives et ses ressorts géographiques. Les notions de frontières et de limites judiciaires relèvent aussi de l'histoire des pratiques à l'instar des recherches anciennes menées par Bernard Schnapper sur la "litigiosité". L'histoire des paysages judiciaires, et notamment des paysages criminels, fait partie intégrante de cette première grande direction. Toutefois, je crois qu'il faudrait aussi revenir, dans la perspective d'une étude de la justice de proximité, sur la notion de "pays". De nouveau d'actualité aujourd'hui, avec une acception différente de celles du passé, le "pays" à la fin du XVIIIème siècle et au début du XIXème siècle, se limite en fait au quartier ou au village. La distance entre pays étant évaluée en coups de fusil. L'inscription de la justice dans un territoire nécessite de rompre avec une vision uniforme de l'espace. A titre d'illustration, soulignons que le territoire des justiciables n'est pas nécessairement celui des administrateurs.

La deuxième grande direction se rapporte moins à un thème qu'à un support, celui de la presse qui représente, à elle seule, plusieurs chantiers, que ce soit les canards d'Ancien Régime ou la presse plus spécialisée au XIXème siècle. Plusieurs mémoires de maîtrise ont été réalisés à 
partir de la Gazette des tribunaux. Périodique judiciaire le plus important du XIXème siècle, miroir de la société à une époque donnée, la Gazette des tribunaux est un véritable univers. Des romanciers illustres, Maupassant, Flaubert, Courteline, vont y puiser des informations et y trouver le point de départ de sujets qui correspondent à une attente du lectorat. Ce périodique judiciaire publie également des comptes-rendus de procès politiques. Par exemple le procès des quinze (1832) ou l'affaire des poudres (1836) relatifs à Auguste Blanqui, révolutionnaire et inlassable conspirateur qui a sacralisé l'insurrection jusqu'à compter le nombre de pavés qui devaient constituer chaque barricade et qui a passé plus de la moitié de sa vie en prison. Toujours est-il que la Gazette a restitué les jugements et retranscrit les débats, les réquisitoires et les plaidoiries. Source pratiquement unique, elle est conservée en partie à Poitiers.

La troisième entrée se rapporte à l'étude de la justice criminelle. Il s'agit de privilégier quelques thèmes ou approches, en particulier la question du témoignage. Il faudrait s'interroger sur la manière dont celui-ci se construit, sur la "fabrique" des faux témoignages, sur le délitement de la preuve testimoniale et sur l'enchevêtrement et le recoupement des témoignages. Tous ceux qui ont travaillé sur un dossier d'instruction savent bien que pour une unique affaire, cinquante témoignages peuvent être recueillis. Quand on les met bout à bout et quand on les confronte et les compare, il devient possible de rendre compte de façon concrète du fonctionnement de la justice et de la perception des "acteurs", protagonistes ou simples observateurs. Ce qui est passionnant pour la recherche, au-delà de l'intérêt que l'on peut porter à une affaire singulière, à un coupable ou à une victime, c'est que c'est l'ensemble d'une société qui est captée dans les rets de la justice. Il faudrait aussi s'attacher, en déconstruisant la taxinomie judiciaire, à d'autres faits criminels, à l'instar d'un excellent mémoire de maitrise qui s'était intéressé au massacre des vieillards. L'analyse a mis en évidence les mécanismes d'occultation d'un phénomène dissimulé par la dénomination des infractions criminelles ; elle a aussi permis de révéler comment se construisait la figure du "vieillard judiciaire" à travers la presse. Enfin, il faudrait traiter de la préméditation. Sur ce vaste sujet, une thèse est en 
préparation. Elle nécessite un effort de reconstruction des affaires criminelles et une interrogation constante sur le passage à l'acte.

Quatrième grande direction que je me contenterai simplement d'énoncer. Dans l'immédiat, les recherches sont peu avancées, il s'agit davantage d'une réflexion liminaire autour de la justice commerciale, parent pauvre des travaux sur la justice. Edouard Bouyé, directeuradjoint des Archives départementales de la Vienne, n'a pas hésité à faire un tour du département afin d'engranger liasses et cartons. Les travaux possibles s'avèrent fort nombreux. Pour ne prendre qu'un seul exemple, soulignons qu'il est possible de faire une histoire des marques de fabrique à partir des sources commerciales.

Une cinquième approche part d'un constat simple. Jusqu'à présent la communauté des chercheurs, spécialistes de l'histoire de la justice, a pratiquement semblé ignorer qu'il existait une justice sexuée. Quelques travaux en cours s'intéressent plus particulièrement aux femmes criminelles et aux femmes victimes, aux pratiques des unes et aux perceptions des autres. Le colloque international de 2001 qui s'est tenu à Angers, organisé par l'Hirés avec la collaboration du Gerhico, sera l'occasion, pour la première fois, de traiter globalement de la question

La cinquième grande direction concerne "l'instruction judiciaire", c'est-à-dire l'investigation judiciaire ou l'enquête judiciaire, et l'expertise judiciaire. Entre la Mission de recherche droit et justice et le Gerhico, une convention a été signée qui a depuis débouché sur la réalisation d'un rapport scientifique relatif à l'histoire de l'expertise judiciaire en France de 1791 à 1944. Dans l'avenir proche, il est envisagé de travailler sur l'erreur judiciaire. A la fin du XIXème siècle, la plupart des magistrats arguent du fait que l'erreur judiciaire ne vient pas des juges, mais des spécialistes qui ont été diligentés par la justice et auxquels on a accordé trop de crédit. Autrement dit, affirment avec force les magistrats, l'erreur judiciaire est une erreur technique.

Enfin, sixième et dernière piste : l'iconographie judiciaire. S'il existe des travaux de très grande qualité sur les "images de la justice" à l'époque médiévale et moderne, le déficit des études pour la période contemporaine est particulièrement criant. 
Ces aspects, rapidement énumérés se veulent des encouragements à poursuivre "l'auscultation judiciaire", pour reprendre une formule des années 1930, à condition toutefois de croiser les regards et les approches, et de multiplier les collaborations. 